\title{
Die chronische hintere Knieinstabilität - ein noch ungelöstes Problem?
}

ährend sich die klinische und wissenschaftliche Aktivität im letzten Jahrzehnt vor allem dem vorderen Kreuzband gewidmet hat, erlangen nun auch die Verletzungen und Verletzungsfolgen der hinteren Kniebandstrukturen zunehmende Bedeutung. Gerade die Therapie chronischer hinterer Knieinstabilitäten gehört dabei zu den anspruchsvollsten Aufgaben in der Kniegelenkschirurgie. Eine adäquate Versorgung dieser Verletzungsfolgen ist an bestimmte Voraussetzungen gebunden: der exakte Instabilitätstyp (isoliert, komplex, posterolateral, posteromedial) sollte zunächst durch spezifische klinische Tests differenziert werden, die Problematik der knöchernen Beinachse und eines eventuellen Varusmorphotyps muß beurteilt werden, der Knorpelstatus sollte in die Planung einbezogen werden und nicht zuletzt sollte anamnestisch sorgfältig ausgelotet werden, ob tatsächlich Instabilitätsbeschwerden für den Patienten im Vordergrund seiner Problematik stehen. Aufwendige operative Rekonstruktionen können arthrosetypische Schmerzen verstärken und sollten daher bei fortgeschrittener Gelenksdegeneration nicht durchgeführt werden.
Während Details des operativen Vorgehens heute noch kontrovers gesehen werden, gibt es doch bereits etablierte Prinzipien der operativen Stabilisierung der hinteren Knieinstabilität. Der anatomische Ersatz des hinteren Kreuzbandes unter Betonung des anterolateralen Hauptbündels erfolgt mit einem freien Bindegewebstransplantat. Patellarsehnentransplantate werden ebenso wie Quadrizepssehnenstreifen und gebündelte Pes anserinus-Sehnen verwendet, ohne daß derzeit klare Vorzüge eines Transplantatlagers erkennbar wären. In der überwiegenden Zahl der Fälle ist zusätzlich zum hinteren Kreuzbandersatz eine Stabilisierung der posterolateralen Gelenkecke mit einem geeigneten Verfahren erforderlich. Diese posterolateralen Stabilisierungen erfordern eine genaue Kenntnis der Anatomie und der Biomechanik des lateralen Seitenbands, des Popliteus und des posterolateralen Gelenkecks. Die Prognose des Eingriffs hängt stark von der Beseitigung der posterolateralen Rotationsinstabilität $a b$, so daß diesem Schritt der Operation besondere Bedeutung zukommt. Bei Vorliegen eines knöchernen Varusmorphotyps sind die Folgen einer Verletzung des posterioren und posterolateralen Bandapparats besonders ausgeprägt, da unter Belastung ständig Distraktionskräfte auf die Verletzungsregion einwirken. Es sollte in diesen Fällen abgewogen werden, ob nicht im er- sten Schritt eine valgisierende und reklinierende interligamentäre Hebeosteotomie durchgeführt werden sollte, um die laterale Distraktion zu beseitigen und damit die Voraussetzung für bandplastische Eingriffe zu verbessern.

Unter Berücksichtigung der schweren Behinderung, welche eine ausgeprägte chronische posteriore und posterolaterale Knieinstabilität für die meist jungen Verletzten bedeutet, erscheint der hohe Aufwand gerechtfertigt, den die operative Therapie dieser Instabilitätsform erfordert. Das Leitthema dieses Heftes soll vor allem die Diagnostik, die Indikationsstellung und die Prinzipien der operativen Therapie darstellen. Der weitere intensive Austausch hinsichtlich der Techniken und vor allem der Ergebnisse wird erforderlich sein, um eine Verbesserung der noch inkonstanten Resultate zu erreichen. Es ist offensichtlich, daß diese komplexe Chirurgie derzeit aufOperateure zentriert werden sollte, welche sich speziell mit dieser Materie beschäftigen.

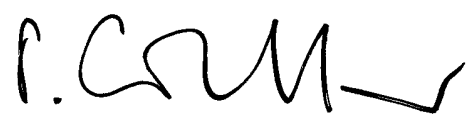

P. Lobenhoffer

Prof. Dr. P. Lobenhoffer Chefarztklinik für Unfall- und Wiederherstellungschirurgie, Henriettenstiftung Hannover, Marienstraße 72-90, D-30171 Hannover 